ARTIGO ORIGINAL

\title{
Uso de simbiótico no tratamento de pacientes obstipados com esclerose lateral amiotrófica
}

\author{
Use of synbiotic in the treatment of constipated patients with amyotrophic lateral sclerosis
}

\author{
Maria Lúcia Oliveira Dourado Siqueira, Júlia Leite Castro², Silvia Helena Nardi Baroni³, \\ Sancha Helena de Lima Vale ${ }^{4}$, Camila Xavier Alves $^{5}$, Márcia Marília Gomes Dantas ${ }^{5}$, \\ Karina Marques Vermeulen ${ }^{6}$, Lúcia Dantas Leite ${ }^{7}$
}

\section{RESUMO}

Introdução: O uso de simbióticos no tratamento da obstipação parece ser promissor, sobretudo em portadores de esclerose lateral amiotrófica (ELA) que apresentam a obstipação como sintoma frequente.

Objetivo: avaliar o efeito da suplementação de simbiótico sobre o funcionamento intestinal de pacientes obstipados com ELA.

Materiais e Métodos: Este estudo de intervenção foi realizado com pacientes acompanhados pelo ambulatório multidisciplinar de ELA do Hospital Universitário Onofre Lopes (HUOL) da Universidade Federal do Rio Grande do Norte (UFRN). O estudo foi composto por três grupos: 10 controles saudáveis (grupo 1); 10 portadores de ELA obstipados (grupo 2); e 10 portadores de ELA não obstipados (grupo 3). Todos os participantes foram suplementados com de simbiótico (6g/dia), e monitorados por um período de 15 dias quanto à função intestinal.

Resultados: Participaram do estudo 30 sujeitos (13 homens e 17 mulheres), com idade média de 54,3 (dp=10,6) anos. No grupo 1 não houve alterações para a maioria dos parâmetros observados. No entanto, nos grupos 2 e 3, observouse melhora no funcionamento intestinal, representada pela presença de evacuação, melhora da consistência das fezes e menor esforço ao evacuar. Essas alterações foram visíveis após 1 semana de suplementação com simbiótico.

Conclusão: O uso regular de simbiótico pode melhorar o funcionamento intestinal em portadores de ELA, estejam eles obstipados ou não.

Palavras-chave: nutrição; esclerose lateral amiotrófica; prebióticos; probióticos; obstipação.

\begin{abstract}
Introduction: The use of synbiotic in the management of constipation seems to be promising, particularly in patients with amyotrophic lateral sclerosis (ALS) which have constipation as a frequent symptom.

Objective: To evaluate the effect of synbiotic supplementation on intestinal function of constipated patients with ALS.

Materials and Methods: This interventional study was performed with ALS patients followed at the ALS multidisciplinary ambulatory of Hospital Universitário Onofre Lopes (HUOL) at the Federal University of Rio Grande do Norte (UFRN). Subjects were arranged in three groups: 10 healthy controls (group 1), 10 ALS constipated patients (group 2), and 10 ALS non-constipated patients (group 3). All subjects received synbiotic supplementation (6g/day) during 15 days and their intestinal function was monitored daily.

Results: We studied 30 subjects (13 male and 17 female) with mean age $54.3 \pm 10.6$ years. Group 1 didn't show major modifications in the parameters observed. Nevertheless, groups 2 and 3 improved the intestinal function, with increased frequency of bowel movements, improved stool consistency and less evacuation effort. These benefits were observed after one-week of synbiotic supplementation.

Conclusion: Regular use of synbiotic can improve intestinal function in both constipated and non-constipated ALS patients.
\end{abstract}

Keywords: nutrition; amyotrophic lateral sclerosis; prebiotics; probiotics; constipation.

\footnotetext{
${ }^{1}$ Nutricionista. Especialista em Nutrição Funcional pela VP Consultoria.

${ }^{2}$ Nutricionista. Especialista em Nutrição Clínica pela Universidade Gama Filho.

${ }^{3}$ Nutricionista. Especialista em Nutrição Clínica Avançada pela Universidade Potiguar.

${ }^{4}$ Nutricionista. Doutora em Ciências da Saúde pela Universidade Federal do Rio Grande do Norte (UFRN).

${ }^{5}$ Nutricionista. Mestre em Ciências da Saúde pela UFRN.

${ }^{6}$ Nutricionista graduada pela UFRN.

${ }^{7}$ Nutricionista. Doutora em Ciências da Saúde pela UFRN. Professora Adjunta do Departamento de Nutrição da UFRN.
} 


\section{INTRODUÇÃO}

A Esclerose Lateral Amiotrófica (ELA) é uma doença neurodegenerativa, progressiva, que acomete os neurônios motores superiores e inferiores. É caracterizada principalmente por fraqueza e atrofia muscular e possui um prognóstico desfavorável1-3. Apesar dos mecanismos fisiopatológicos da ELA serem intensamente pesquisados, sua etiologia ainda é desconhecida. Acredita-se em hipóteses etiopatogênicas incluindo estresse oxidativo, inflamação, excitotoxicidade do glutamato, disfunção mitocondrial, agregação proteica, defeito no processamento de RNA, distúrbio no metabolismo de metais e efeitos tóxicos de metais pesados ${ }^{3}$. Apesar das hipóteses existentes, nenhum fator isolado é suficiente para explicar todas as formas da doença ${ }^{4-6}$. A forma da ELA familiar abrange 5 a $10 \%$ dos casos, possui causa genética e tem início precoce em relação a ELA esporádica. Entretanto não há distinções clínicas entre essas formas ${ }^{7-9}$.

O diagnóstico da ELA é clínico, podendo ser complementado pela eletroneuromiografia e estudos de neuroimagem ${ }^{10,11}$. Os critérios diagnósticos utilizados permitem classificar a ELA em quatro níveis: suspeita, possível, provável e definitiva ${ }^{12}$. A ELA tem uma incidência mundial em torno de 1,0 a 2,5 casos/100.000 habitantes ${ }^{13}$, aumentando para 7,5 casos/100.000 habitantes na população acima dos 80 anos $^{14}$. Algumas peculiaridades descritas na literatura despertam interesse quanto à alta incidência verificada na ilha de Guam e na Nova Guiné ${ }^{15,16}$. Sua prevalência mundial apresenta variações entre 4 a 6/100.000 habitantes ${ }^{17}$.

A disfagia e a obstipação são sintomas comuns em pacientes com $E L A^{18}$. O baixo consumo alimentar associado ao aumento do gasto energético e ao prejuízo na absorção intestinal de nutrientes contribuem para a desnutrição e para menor sobrevida ${ }^{19-25}$. A obstipação, por si só, já é um incômodo encontrado frequentemente na população idosa ${ }^{26}$ e nos países ocidentais ${ }^{27}$. Na ELA, por sua vez, a obstipação não é um sintoma neurológico primário, mas está presente em $57 \%$ dos pacientes. $\mathrm{Na}$ literatura, a obstipação é caracterizada por número de evacuações reduzidas $(<3 x /$ sem $)$, relato de fezes ressecadas, dor ao evacuar ou sensação de evacuação incompleta 27,28 .
Nesse contexto, os probióticos, prebióticos e simbióticos têm sido utilizados no tratamento da obstipação devido aos seus efeitos positivos sobre a integridade da mucosa, o peristaltismo e a ação imunomodulatória ${ }^{29}$. Define-se probióticos como microrganismos que, quando ingeridos em quantidade adequada, exercem benefícios ao hospedeiro por alterar satisfatoriamente a flora intestinal. Atualmente existe uma gama de produtos comercializados contendo microrganismos probióticos. Os mais comuns são lactobacilos, bifidobactérias e leveduras não patogênicas. Já os prebióticos, são definidos como substâncias não digeríveis, mas fermentáveis em nível intestinal, capazes de beneficiar o hospedeiro por estimular seletivamente o crescimento e a atividade de bactérias benéficas que residem no cólon. Os frutooligossacarídeos (FOS) e a inulina são os prebióticos mais comuns. Os simbióticos, por sua vez, é o termo usado para definir um produto que contém tanto probióticos como prebióticos, objetivando a ação sinérgica de melhorar a quantidade e a qualidade da microflora intestinal do hospedeiro ${ }^{29,30}$.

Verifica-se a escassez de estudos que avaliam o efeito dos simbióticos sobre a obstipação secundária em pacientes neurológicos ${ }^{31}$. Diante disso, justifica-se a realização desse estudo de intervenção, cujo objetivo foi avaliar o efeito da suplementação de simbiótico sobre o funcionamento intestinal de pacientes obstipados com ELA.

\section{MATERIAIS E MÉTODOS}

\section{Delineamento e amostra}

O presente estudo transversal de intervenção foi realizado com os portadores de ELA, acompanhados pelo ambulatório multidisciplinar de ELA do Hospital Universitário Onofre Lopes (HUOL), da Universidade Federal do Rio Grande do Norte (UFRN). Além dos pacientes, um grupo controle com indivíduos saudáveis também foi incluído. Esse estudo foi aprovado pelo Comitê de Ética em Pesquisa do HUOL/UFRN, mediante protocolo no 436/10. Todos os participantes ou responsáveis legais assinaram o Termo de Consentimento Livre e Esclarecido (TCLE). Dentre os 


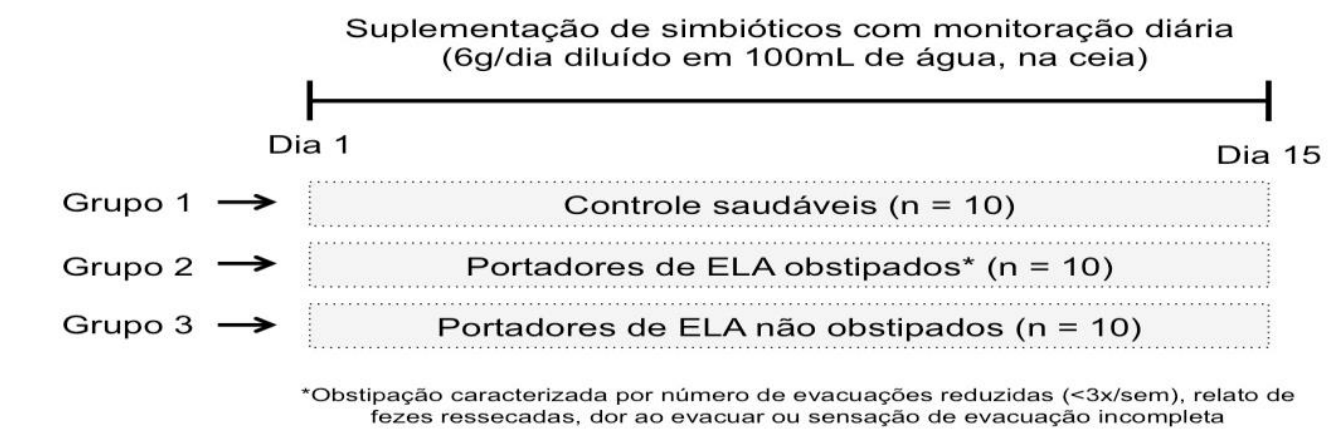

FIGURA 1 - Delineamento do estudo.

pacientes, foram incluídos aqueles em dieta oral ou enteral, com funcionamento intestinal satisfatório ou com obstipação. Considerou-se como funcionamento intestinal satisfatório a ausência de diarreia (três ou mais evacuações líquidas por dia) e obstipação (menos de três evacuações por semana, fezes ressecadas, dor ao evacuar ou sensação de evacuação incompleta) $)^{27,28}$. Foram excluídos do estudo aqueles em uso de medicamentos laxantes, antidiarreicos, antibióticos ou qualquer outro que interferisse na motilidade intestinal, aqueles em uso de suplementos e/ou módulos de fibras e aqueles com síndromes disabsortivas. Os indivíduos saudáveis, por sua vez, foram selecionados por conveniência, obedecendo aos critérios de exclusão dos pacientes.

Os pacientes foram divididos em três grupos: 10 controle saudáveis (grupo 1); 10 portadores de ELA obstipados (grupo 2); e 10 portadores de ELA não obstipados (grupo 3). Todos os participantes foram suplementados com produto simbiótico de marca registrada, e monitorados por um período de 15 dias (figura 1).

\section{Coleta dos dados}

As informações necessárias para a caracterização dos pacientes e do funcionamento intestinal foram coletadas mediante preenchimento de um formulário específico em entrevistas realizadas com os participantes ou seus respectivos cuidadores. Essas entrevistas ocorreram durante as consultas ambulatoriais, em visitas domiciliares ou mediante contato telefônico diário.

\section{Suplementação de simbiótico}

Os participantes do estudo foram suplementados durante 15 dias consecutivos com um sachê $(6 \mathrm{~g})$ por dia de um produto simbiótico comercialmente disponível, diluído em $100 \mathrm{~mL}$ de água, sendo ofertado no horário da ceia. Esse produto apresenta em sua composição cepas probióticas de Lactobacillus paracasei, Lactobacillus acidophillus, Lactobacillus rhamnosus e Bifidobactrium lactis, nas quantidades de 109 a 108 UFC para cada cepa. Além disso, contém frutooligossacarídeo (FOS), na quantidade de $6 \mathrm{~g}$, correspondendo ao componente prebiótico.

\section{Análise estatística}

Os dados foram analisados mediante estatística descritiva de tendência central e dispersão, e foram apresentados em tabela e gráfico, com auxílio do GraphPad Prism 6.0.

\section{RESULTADOS}

O estudo foi composto por 30 participantes distribuídos equitativamente no grupo 1 (controle saudáveis), no grupo 2 (portadores de ELA obstipados) e no grupo 3 (portadores de ELA não obstipados). No geral, participaram 13 homens e 17 mulheres, com idade média de 54,3 $\pm 10,6$ anos. Quanto ao diagnóstico classificatório de ELA, $100 \%$ dos participantes do grupo 2 tinham ELA definitiva, enquanto que no grupo 3, 30\% apresentavam ELA provável, 60\% ELA definitiva e apenas $10 \%$ ELA possível. Em relação ao tempo de doença após o diagnóstico, verificou-se que a mediana 
TABELA 1 - Comparação do funcionamento intestinal dos participantes entre o baseline (dia 0) e o último dia do estudo (dia 15).

\begin{tabular}{|c|c|c|c|c|c|c|c|}
\hline \multirow[t]{2}{*}{ Parâmetros } & \multirow[t]{2}{*}{ Informação } & \multicolumn{2}{|c|}{ Grupo $1(\%)$} & \multicolumn{2}{|c|}{ Grupo $2(\%)$} & \multicolumn{2}{|c|}{ Grupo $3(\%)$} \\
\hline & & DIA 0 & DIA 15 & DIA 0 & DIA 15 & DIA 0 & DIA 15 \\
\hline \multirow{2}{*}{$\begin{array}{l}\text { Presença de } \\
\text { evacuação }\end{array}$} & Sim & 80 & 80 & 10 & 50 & 70 & 100 \\
\hline & Não & 20 & 20 & 90 & 50 & 30 & - \\
\hline \multirow{2}{*}{$\begin{array}{l}\text { Frequência de } \\
\text { evacuações }\end{array}$} & 1 a $3 x / d i a$ & 80 & 80 & 10 & 50 & 60 & 100 \\
\hline & $>3 x / d i a$ & - & - & - & - & 10 & - \\
\hline \multirow{6}{*}{ Consistência das fezes } & Sólida & - & - & - & 30 & 50 & 70 \\
\hline & Caprina & - & 10 & - & 10 & - & - \\
\hline & Ressecada & 40 & - & 10 & - & - & - \\
\hline & Pastosa & 20 & 60 & - & 10 & 10 & 20 \\
\hline & Semilíquida & 20 & 10 & - & - & - & - \\
\hline & Líquida & - & - & - & - & 10 & 10 \\
\hline \multirow{3}{*}{ Volume das fezes } & Grande & 10 & - & - & - & 10 & 30 \\
\hline & Médio & 70 & 80 & - & 30 & 60 & 70 \\
\hline & Pequeno & - & - & 10 & 20 & - & - \\
\hline \multirow{4}{*}{$\begin{array}{l}\text { Sintomas } \\
\text { gastrintestinais }\end{array}$} & Flatulência & - & - & 10 & 20 & 10 & 20 \\
\hline & Empachamento & - & - & - & - & - & - \\
\hline & Distensão abdominal & - & - & 10 & - & - & - \\
\hline & Cólica & 10 & - & - & - & 10 & - \\
\hline \multirow{4}{*}{ Esforço ao evacuar } & Sem esforço & 80 & 80 & - & 40 & 50 & 100 \\
\hline & Leve & - & - & - & 10 & 20 & - \\
\hline & Moderado & - & - & - & - & - & - \\
\hline & Grande & - & - & 10 & - & - & - \\
\hline \multirow{2}{*}{ Dor ao evacuar } & Ausente & 70 & 80 & 10 & 50 & 60 & 100 \\
\hline & Presente & 10 & - & - & - & 10 & - \\
\hline
\end{tabular}

(mínimo - máximo) para o grupo 2 foi de $2,5(0,1$ - 10,5) anos, e para o grupo 3 foi de 2,3 $(0,2-6,6)$ anos. Os pacientes estudados se alimentavam pela via oral, exceto 1 (10\%) paciente do grupo 2.

As informações sobre o funcionamento intestinal dos pacientes, coletadas no baseline do estudo ou dia 0 , foram usadas para criação do grupo 2 (obstipados) e grupo 3 (não obstipados), e serviram como parâmetro inicial para comparação do efeito da suplementação de simbiótico (tabela 1).

Comparando-se o dia 0 com o dia 15, observamos que houve modificações no perfil do funcionamento intestinal entre os grupos estudados. No grupo 1 não houve alterações para a maioria dos parâmetros observados. No entanto, observou-se melhora na consistência das fezes, saindo de uma maior representatividade de fezes ressecadas (40\%) para fezes pastosas $(60 \%)$ (tabela 1$)$. Tanto no grupo 2 quanto no grupo 3, observou-se melhora quanto a presença de evacuação e a consistência das fezes, além de menor esforço ao evacuar. Ainda quanto à presença de evacuações, houve melhora para os grupos 2 e 3 principalmente na primeira semana do estudo, com estabilização desse parâmetro na segunda semana. No grupo 1, controle saudável, observou-se apenas manutenção quanto a presença das evacuações, comprovado pela linha praticamente constante (figura 2).

\section{DISCUSSÃo}

No presente estudo demonstramos que a administração do simbiótico comercial melhorou a 
função intestinal dos portadores de ELA. Não encontramos registro na literatura de outro trabalho que tenha avaliado o uso de simbiótico nesses pacientes. Sabemos que a obstipação é frequente na ELA por vários motivos, tais como: baixa ingestão hídrica, desidratação, baixa consumo de fibras, uso de medicamentos anticolinérgicos e opióides, além da imobilidade progressiva decorrente da própria da doença ${ }^{24,25}$. Apesar da ausência de estudos publicados nessa temática, benefícios do uso de prebióticos, probióticos ou simbióticos têm sido demonstrados em pacientes com algum comprometimento neurológico.

Um estudo piloto em indivíduos com Doença de Parkinson demonstrou que o uso regular de probióticos melhora significativamente a obstipação ${ }^{32}$. Em adição, uma meta-análise avaliou o efeito do uso de prebióticos, probióticos e simbióticos sobre a modulação da flora intestinal em pacientes com encefalopatia hepática. Em dois estudos incluídos nessa meta-análise, o uso de probióticos e simbióticos tiveram efeitos benéficos e significativos. Além disso, essas substâncias não causaram maiores efeitos adversos e foram melhor toleradas em comparação a lactulose, muitas vezes utilizada no tratamento da obstipação ${ }^{33}$.

No nosso estudo, apenas $10 \%$ dos pacientes com ELA obstipados (grupo 2) estavam com evacuação presente no baseline do estudo (dia 0 ). No final do estudo (dia 15), esse percentual aumentou para $50 \%$. Em consonância, também houve melhora do funcionamento intestinal nos pacientes com ELA não obstipados (grupo 3), uma vez que o percentual de pacientes com evacuação presente aumentou de $70 \%$ (dia 0) para $100 \%$ (dia 15). Além disso, ao final do estudo, $100 \%$ desses pacientes não apresentavam dor ao evacuar. Esse dado demonstrou benefício do uso do simbiótico no funcionamento intestinal dos portadores de ELA, independente de serem obstipados ou não.

Os benefícios do uso de prebióticos, probióticos e simbióticos extrapola um grupo específico de pacientes, pois seu efeito terapêutico não está relacionado ao tratamento de uma patologia específica, mas sintomas decorrentes do prejuízo funcional do intestino. Diante disso, a aplicação dessas substâncias na prática clínica tem sido ampla, independente do sexo, da faixa etária

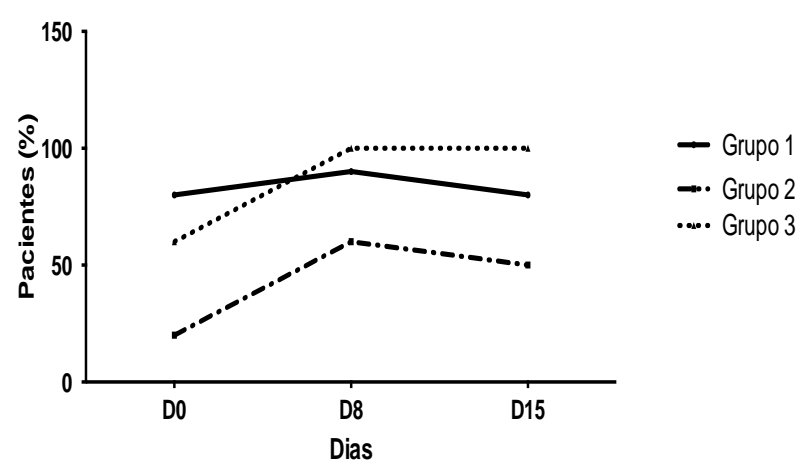

FIGURA 2 - Distribuição percentual dos pacientes quanto a presença de evacuação.

ou da presença de enfermidades ${ }^{34}$.

Amenta et al. ${ }^{27}$ avaliaram o efeito da suplementação de simbiótico (Bifidobacterium longum W11 + FOS Actilight) em 297 pacientes obstipados, submetidos à dietas hipocalóricas para perda de peso. Após 20 dias de suplementação, esses autores observaram efeito benéfico, comprovado pela melhora do quadro. Em outro estudo, mulheres obstipadas tiveram melhora significativa da função intestinal com o consumo regular de iogurte com simbióticos ${ }^{35}$. Em consonância, Paineau et al. ${ }^{36}$ demonstraram em idosos um aumento da frequência evacuatória após suplementação de simbiótico, contendo Bifidobacterium animalis e oligofrutose enriquecida com inulina, contribuindo indiretamente para o bem estar e a qualidade de vida dessa população.

Em artigo original, Vitali et al. ${ }^{37}$ estudaram o impacto da suplementação mensal de simbiótico sobre a composição microbiota e o perfil metabólico, em 20 adultos com idade entre 20 e 50 anos. Esses autores verificaram que essa intervenção promoveu modulação das atividades metabólicas intestinais, com aumento significativo de ácidos graxos de cadeia curta, cetonas, dissulfeto de carbono e acetato de metila, sugerindo promoção à saúde intestinal. Uma publicação recente de revisão sistemática selecionou cinco estudos randomizados com um total de 377 indivíduos, adultos e crianças, com obstipação tratados com probióticos. Os dados mostraram benefícios de algumas cepas e outras não. Os autores concluíram que o uso de probióticos para obstipação necessita de mais investigações ${ }^{31}$.

Atualmente é fato o benefício do uso de simbióticos em situações como doenças diarreicas, doenças 
inflamatórias intestinais, síndrome do intestino irritável, desordens infecciosas, obstipação ${ }^{30}$, prevenção de reações alérgicas ${ }^{29}$ e modulação da resposta imunológica ${ }^{38}$. No entanto, sabe-se que com o avançar da idade há grande alteração da flora intestinal, representada por diminuição de bifidobactérias e aumento de enterobactérias. Essas alterações são maximizadas não apenas pelo avançar da idade, mas principalmente por modificações na dieta, no estado nutricional, por presença de doenças e uso de medicações. Nesse sentido, indivíduos idosos doentes ou não, neurológicos ou não, podem se beneficiar com uso de probióticos, prebióticos e simbióticos ${ }^{39}$.

Muitas foram as limitações do nosso estudo. A raridade da doença restringiu o tamanho amostral, limitando a análise dos dados à estatística descritiva. $A$ ausência de estudos sobre uso de simbióticos em pacientes com ELA enfocando a temática abordada foi um obstáculo para discussão dos resultados encontrados. Diante disso, considerando os importantes efeitos dos prebióticos, probióticos e simbióticos para a saúde humana, sugere-se que mais estudos sejam feitos mostrando seus benefícios em pacientes neurológicos, sobretudo com ELA. Por fim, concluímos que o uso de simbiótico melhorou o funcionamento intestinal de portadores de ELA, obstipados ou não, mediante da melhora na presença de evacuação e consistência das fezes.

\section{REFERÊNCIAS}

1. Sabel CE, Boyle PJ, Löytönen M, Gatrell AC, Jokelainen M, Flowerdew R, Maasilta P. Spatial clustering of Amyotrophic Sclerosis in Finland at place of birth and place of death. Am J Epidemiol. 2003;157:898-905.

2. Okamoto K, Kobashi G, Washio M, Sasaki S, Yokoyama T, Miyake Y, Sakamoto N, Tanaka H, Inaba Y. Descriptive epidemiology of Amyotrophic Lateral Sclerosis in Japan, 1995-2001. J Epidemiol. 2005;15:20-3.

3. Barber SC, Shaw PJ. Oxidative stress in ALS: Key role in motor neuron injury and therapeutic target. Free Radic Biol Med. 2010; 48:629-41.

4. Chiappetta ALML. Disfagia orofaríngea em pacientes com doença do neurônio motor: esclerose lateral amiotrófica [tese]. São Paulo (SP): UNIFESP/EPM; 2005.

5. Silva HCA. Etiopatogenia da ELA: causa única ou várias causas? Rev Neurocienc. 2006;14(2):34-42.

6. Werneck LC, Bezerra R, Silveira Neto O, Scola RH. A clinical epidemiological study of 251 cases of Amyotrophic Lateral Sclerosis in the south of Brazil. Arq Neuropsiquiatr. 2007;65(2):189-95.
7. Aguirre T, Matthijs G, Robberecht W, Tilkin P, Cassiman JJ. Mutational analysis of the $\mathrm{Cu} / \mathrm{Zn}$ superoxide dismutase gene in 23familial and 69 sporadic cases of amyotrophic lateral sclerosis in Belgium. Eur J Hum Genet. 1999; 7:599-602.

8. Castro-Costa CM, Oriá RB, Vale OC, Arruda JAM, Horta WG, D'almeida JAC, Santos TJT, Ramos RSN, Gifoni MAC. Motor neuron diseases in the University Hospital of Fortaleza (Northeastern Brazil): a clinico-demographic analysis of 87 cases. Arq Neuropsiquiatr. 2000;58:986-9.

9. Tagawa A, Tan CF, Kikugawa K, Fukase M, Nakano R, Onodera O, Nishizawa M, Takahashi $H$. Familia amyotrophic lateral sclerosis: a SOD1-unrelated japanese Family of bulbar type with Bunina bodies and ubiquitinpositive skein-like inclusions in lower motor neurons. Acta Neuropathol. 2007; 113:205-11.

10. Dourado Junior MET. Eletroneuromiografia: Estimativa do número de unidades motoras na ELA. Rev Neuroc. 2006; 14(2):24-34.

11. Le Forestier N. Diagnostics différentiels de la sclérose latérale amyotrophique. The differential diagnosis of amyotrophic lateral sclerosis. Rev Med Liege. 2004; 59(1):25-31.

12. Brooks BR, Miller RG, Swash M, Munsat TL. El Escorial revisited: revised criteria for the diagnosis of amyotrophic lateral sclerosis. ALS other Motor Neuron Disord. 2000;1:293-9.

13. Logroscino G, Beghi E, Zoccolella S, Palagano R, Fraddosio A, Simone IL, Lamberti P, Lepore V, Serlenga L. Incidence of Amyotrophic Lateral Sclerosis in southern Italy: a population based study. J Neurol Neurosurg Psychiatry. 2005;76(8):1094-8.

14. Forbes RB, Colville S, Parratt J, Swingler RJ. The incidence of motor neuron disease in Scotland. J Neurol. 2007; 254:866-9.

15. Poorkaj P, Tsuang D, Wijsman E, Steinbart E, Garruto RM, Craig UK, Chapman NH, Anderson L, Bird TD, Plato CC, Perl DP, WeiderholT W, Galasko D, Schellenberg GD. TAU as a susceptibility gene for amyotrophic lateral sclerosis - parkinsonism dementia complex of Guam. Arch Neurol. 2001; 58(11):1871-8.

16. Pereira RDB. Epidemiologia: ELA no mundo. Rev Neuroc. 2006; 14(2):9-13.

17. Matias-Guiu J, Galán L, Garcia-Ramos R, Vela A, Guerrero A. Epidemiología descriptiva de La esclerosis lateral amiotrófica. Neurologia. 2007;22(6):368-80.

18. Rodriguez GE, Waisman MIR, Duek V, Sica REP. Evaluación fonodeglutitória en pacientes con esclerosis lateral amiotrófica. Prensa Med Argent. 2005; 92:236-48.

19. Vaisman N, Lusaus M, Nefussy B, Niv E, Comaneshter D, Hallack R, Drory VE. Do patients with amyotrophic lateral sclerosis (ALS) have energy needs? J Neurol Sci. 2009;279(1-2):26-9.

20. Bouteloup C, Desport JC, Clavelou P, Guy N, DerumeauxBurel H, Ferrier A, Couratier P. Hypermetabolism in ALS patients: an early and persistent phenomenon. J Neurol. 2009;256:1236-42.

21. Silva LB, Mourão LF, Silva AA, Lima NMFV, Almeida SR, Franca Jr MC, Nucci A, Amaya-Farfán J. Amyotrophic lateral sclerosis: combined nutritional, respiratory and functional assessment. Arq Neuropsiquiatr. 2008; 66(2B):354-9.

22. Kasarskis EJ, Berryman S, Vanderleest JG, Schneider AR, Mcclain CJ. Nutritional status of patients with amyotrophic lateral sclerosis: relation to the proximity of death. Am J Clin Nutr. 1996; 63(1):130-7.

23. Desport JCP, Preux PM, Truong TC, Vallat JM, Sautereau 
$D$, Couratier P. Nutritional status is a prognostic factor for survival in ALS patients. Neurology. 1999;53:1059-63.

24. Winge $K$, Rasmussen D, Werdelin LM. Constipation in neurological diseases. J Neurol Neurosurg Psychiatry. 2003;74:13-9.

25. Rocha JA, Reis C, Simões F, Fonseca J, Mendes Ribeiro $J$. Diagnostic investigation and multidisciplinary management in motor neuron disease. J Neurol. 2005; 252(12):1435-47.

26. Kleessen B, Sykura B, Zunft HJ, Blaut M. Effects of inulin and lactose on fecal microflora, microbial activity, and bowel habit in elderly constipated persons. Am J Clin Nutr. 1997;65(5):1397-402.

27. Amenta M, Cascio MT, Di Fiore P, Venturini I. Diet and chronic constipation: benefits of oral supplementation with symbiotic zir fos (Bifidobacterium longum W11 + FOS Actilight). Acta Biomed. 2006; 77(3):157-62.

28. Brandt LJ, Prather CM, Quigley EM, Schiller LR, Schoenfeld $P$, Talley NJ. Systematic review on the management of chronic constipation in North America. Am J Gastroenterol. 2005; 100(1):S5-S21.

29. Gourbeyre P, Denery S, Bodinier M. Probiotics, prebiotics, and synbiotics: impact on the gut immune system and allergic reactions. J Leukoc Biol. 2011;89(5):685-95.

30. Quigley EM. Prebiotics and probiotics: modifying and mining the microbiota. Pharmacol Res. 2010;61(3):213-8.

31. Chmielewska A, Szajewska H. Systematic review of randomized controlled trials: probiotics for functional constipation. World J Gastroenterol. 2010; 16(1):69-75.

32. Cassani E, Privitera G, Pezzoli G, Pusani C, Madio C, Iorio $L$, Barichella $M$. Use of probiotics for the treatment of constipation in Parkinson's disease patients. Minerva Gastroenterol Dietol. 2011;57(2):117-21.

33. Shukla S, Shukla A, Mehboob S, Guha S. Meta-analysis: the effects of gut flora modulation using prebiotics, probiotics and synbiotics on minimal hepatic encephalopathy. Aliment Pharmacol Ther. 2011;33(6):66271.

34. Kolida S, Gibson GR. Synbiotics in health and disease. Annu Rev Food Sci Technol. 2011;2:373-93.

35. De Paula JA, Carmuega E, Weill R. Effect of the ingestion of a symbiotic yogurt on the bowel habits of women with functional constipation. Acta Gastroenterol Latinoam. 2008;38:16-25.

36. Paineau D, Payen F, Panserieu S, Coulombier G, Sobaszek A, Lartigau I, Brabet M, Galmiche JP, Tripodi D, Sacher-Huvelin S, Chapalain V, Zourabichvili O, Respondek F, Wagner A, Bornet FR. The effects of regular consumption of shortchain fructo-oligosaccharides on digestive comport of subjects with minor functional bowel disorders. Brit J Nutr. 2008;13:311-8.

37. Vitali B, Ndagijimana M, Cruciani F, Carnevali $P$, Candela $M$, Guerzoni ME, Brigidi P. Impact of a symbiotic food on the gut microbial ecology and metabolic profiles. BMC Microbiology. 2010;10:1-13.

38. Romeo J, Nova E, Wärnberg J, Gómez-Matínez S, Díaz Ligia LE, Marcos A. Immunomodulatory effect of fibres, probiotics and synbiotics in diferente life-stages. Nutr Hosp. 2010;25(3):341-9.

39. Tiihonen K, Ouwehand AC, Rautonen N. Human intestinal microbiota and healthy ageing. Ageing Res Rev. 2009; 9(2):107-16.

\section{Endereço para correspondência}

Lúcia Dantas Leite

Campus Universitário, Lagoa Nova

Natal/RN - CEP 59078-900

Telefone: +558433422291

E-mail: ludl10@hotmail.com 